\title{
Patent Analysis for the Formulation of Technology Policy: Evidence from 4G LTE Industry
}

\author{
Chiang, Cheng-hsin \\ Macau University of Science \\ and Technology \\ chschiang@must.edu.mo
}

\author{
Liaw, Yi-ching \\ Ming Chi University of \\ Technology \\ ycliaw@mail.mcut.edu.tw
}

\author{
Wu, Rung-tai \\ Nanfang College of Sun Yat-sen \\ University \\ rungtai@mail.nfu.edu.cn
}

\begin{abstract}
Policy-makers seek a more rigorous method of selecting potentially successful technologies to fulfil the requirements of different stakeholders. Patent analysis should be able to assist policy-makers in (1) understanding the development trajectory of technologies and monitoring the status of technological development to gain a dynamic view of the current competition situation; (2) applying the concept of relative patent advantage (RPA) to grasp the comparative advantages or disadvantages of specific technology domains in each nation; and (3) combining the patent data and multivariate methods of analysis to clarify the current state of an industry's leading technologies. With the goal of combining the methods of patent data analysis and multivariate analysis, we assess the 4G LTE techniques and explore the comparative technological advantages of Top 10 countries with most patents. This study aims to provide suggestions to serve as an important reference for each nation in formulating its future technology policies.
\end{abstract}

\section{Introduction}

Given recent increases in international competition, the government of each nation employs technology policy formulation and implementation to guide the future direction of national R\&D, innovation and the upgrading of industrial technologies to enhance national competitiveness [1, 2]. Government support for $R \& D$ has always been essential for the process of enhancing national competitiveness for several reasons. First, the cost of the required resources for $R \& D$ activities is tremendous, and the amount of risks and uncertainties involved greatly exceeds that which a single enterprise can afford [3, 4]. Second, technological innovation depends not only on the involvement of companies and academic research institutions in R\&D activities but also on government investment in infrastructure $[5,6]$. Finally, innovation outcomes raise the level of national competitiveness through various effects, such as knowledge spillover, market spillover and network spillover [7]. Therefore, policy-makers often employ a variety of policy tools, such as technology transfer [4], patent licensing [8], collaborative $R \& D$, tax incentives, $R \& D$ subsidies, industry-academia joint programs or low-interest loans, to provide the resources that companies need to perform $\mathrm{R} \& \mathrm{D}$, to share risks and to increase their willingness to invest in innovative activities, which guide the direction of future industrial development $[9,10]$. Therefore, we know that the formulation and implementation of technology policies by the government is a critical factor in determining future national competitiveness.

However, before considering the formulation of technology policy, we must clarify the issue of how to find sufficient evidence to serve as a basis for decision making [11]. Policy-makers must have a more rigorous tool with which to conceive related issues concerning future technology policies, such as emerging technology screening, technology resource allocation and the analysis of international competition. This analytic process can help to reveal internal and external factors related to the challenges that are encountered and to provide information that is required for the formulation of technology policies, with which stakeholder consensus concerning future development of the nation may be solidified [12].

To support the formulation of technology policy, many researchers attempt to provide methodologies from different perspectives to inform the government. First, expert opinions can be used as a screeningbased research method. For example, researchers may use the Delphi, in-depth interview and scenario analysis methods to collect expert opinions and aggregate the views of technology development trends by interviewees. This type of analysis will also assist in predicting which technology domains have the potential to establish the future technology development direction of a nation [13, 14]. Many researchers also perform bibliometric analysis to identify current research directions and popular 
academic issues by reviewing the status of publications in technology literature (e.g., the number of publications or citations). This method can be employed to predict which technologies may be important in the future $[15,16]$. Finally, using databases, researchers can conduct technical patent research based on such search criteria as the patent title, International Patent Classification (IPC), inventor, assignee, abstract or claim. By investigating the status of patent applications, we can understand the evolutionary trajectory and development status of specific technology, which can serve as an important basis for the formulation of technology policies [17].

Many of the methods for supporting decision making not only assist us in clarifying various internal and external factors that affect technology policy formulation but also provide policy-makers with different perspectives regarding the development of technology policy. However, different decision-making methods are associated with unique assumptions, characteristics, limitations and applicable situations. Therefore, policy-makers must select the most appropriate method(s) based on the actual conditions that they encounter to obtain the information that they need to enhance the quality of decision making with respect to technology policy [9, $18]$.

This study attempted to combine the patent data and multivariate analysis methods to construct a new technology monitoring system that will assist policymakers in clarifying current technology development trends, emergent technologies and the comparative technological advantages of their home country and/or other countries; providing sufficient reliable and accurate evidence for formulating technology policy; and assisting policy-makers in identifying the appropriate direction for the formulation of technology policy. Therefore, this study posed the following question: "How does one construct a decision support system that integrates the patent data and multivariate methods of analysis to provide policy-makers with the information that is necessary for decision making?" To investigate our research question, we obtained the necessary patent data from the United States Patent Database (USPTO), and we applied multidimensional scaling (MDS) to construct a new decision support system. In this study, we used 4G LTE industry as an example for discussion, and we explored the development trajectories and comparative advantages/disadvantages of $4 \mathrm{G}$ LTE technology in United States, Sweden, China, Japan, Korea, Canada, Denmark, Finland, France, and Taiwan as a reference for the government formulation of technology policy.

\section{Literature review}

\subsection{An overview of government-sponsored R\&D projects}

In the context of intensifying international competition, a nation's investment in innovation is critical and indispensable for several reasons $[1,19]$. The development of cutting-edge technology requires considerable resources to yield breakthrough developments that lead to technological innovation. Governments often apply policy tools such as technology transfer, collaborative R\&D, R\&D subsidies, R\&D alliances or industry-academic cooperation to assist companies in proceeding with innovative activities that enable industry technologies to be improved and updated to enhance national competitiveness [12]. The government can use various policy tools to support the implementation of innovation activities and to share the burden of risks and uncertainties in the R\&D process [20,21].

In the $R \& D$ process, academic/research institutions and industry, government all need to take efforts to establish transportation systems, communications technology, power supply, intellectual property protection and other infrastructure. Mutual interaction among governments, academic and research institutions and industry promotes the production of new knowledge, which leads to technological applications and spillover effects as well as accelerates the pace of innovation [5]. The spillover effects (e.g., market, knowledge and network effects) are typically generated by government-sponsored R\&D activities, and such effects result in the spreading of outputs and outcomes throughout the industry [10]. This process encourages the improvement of industrial technologies as well as enhanced economic growth, national competitiveness and social welfare, and it results in positive effects on national development.

In the formulation of technology policy, the government should determine how to acquire sufficient evidence to inform decision making. Technology itself possesses the characteristics of indeterminacy, uncertainty, implicitness and unpredictability, and it lacks a historical trajectory for reference. It is difficult to clarify the internal and external factors that influence national technology development, which significantly increases the complexity involved in formulating policy [22, 23]. During the process of formulating policies, many stakeholders will express their needs to policymakers to attempt to persuade them to include relevant requirements in the formulation of technology policy. With limited technology research budgets, the technology policy expectations of some 
stakeholders will be fulfilled, and conflicts of interests will be difficult to avoid. To reconcile conflicting interests, policy-makers must have a set of rigorous tools for contemplating relevant issues regarding future national technology development [24]. Policy-makers require a set of reasonable decision support systems that enable them to obtain reliable information as evidence that they can generate maximum benefits. Therefore, the issue of how to obtain the variety of information that is necessary for the formulation of technology policy via the constructed decision support system requires in-depth discussion $[25,26]$.

\subsection{Decision support methods for technology policy}

To support the formulation of technology policy, researchers have proposed various informationgathering methods aimed at clarifying internal and external factors that may affect future technology development as a reference for policy-makers in formulating technology policy. Based on a review of previous literature, we classified the decision support methods for technology into three categories: expert opinion basis, bibliometric analysis and patent analysis. In the expert opinion category, researchers collect expert opinions via in-depth interviews, such as Delphi method or scenario analysis. The process of aggregating opinions relies heavily on the understanding, knowledge, experience, intuition and imagination of experts from the technology domain to predict how various elements, including society, economy, culture, legislature and infrastructure, affect technological development. The process then involves investigating specific internal factors (strengths and weaknesses) and external factors (opportunities and threats) that influence future national technology development. From a subjective perspective, this process predicts and traces the trajectory of technological evolution, the status of technology development and future trends, and is a reference for those who are developing technology policy $[13,14]$.

Researchers review related literature to know the trajectory of technology development and emerging technological issues, and forecast possible future research directions as an important foundation for formulating technology policy $[11,26]$. Researchers employ bibliometric indicators in the analysis of scholarly literature, such as the use of the number of published papers as an indicator of the development of a particular technology domain. Bibliometric analysis provide policy-makers knowledge of technology development trends, and these insights can formulate technology policy in the future.
Patents also constitute an important source of information for policy-makers. Through patent database search, policy-makers understand the current status of patent applications and approvals within technology domains. The information provides objective evidence to formulate technology policy, indicating future technological development [27]. Patents provide quantitative information for policy makers, includes the objective, reproducible and valid data to support the decision making process [28]. Researchers applied patent analysis to identify environmental technologies to support decision making [29]. Patent portfolio analysis supports policy-makers to clarify the comparative advantages of technological development between themselves and their competitors. Policy-makers can know how to create future benefits, avoid patent litigation, monitor technological development, ensure R\&D rights and establish competitive advantages [17, 30].

Different forecasting methods provide us with diverse perspectives to consider in the formulation of technology policy and provide necessary information for decision-makers [9, 18]. For policy-makers, it is insufficient to simply grasp assumptions, usages, limitations, applicable timing, advantages, disadvantages and operating procedures for each method of analysis. It is also important to be able to select the most appropriate method for clarifying internal and external factors related to technology policy under various circumstances.

Prior study applied data mining approach to construct technological map, representing the practical application and new method to enhance the effectiveness of technological map [31]. In this study, we aim to revisit the role of patent analysis in support of the formulation of technology policy. We combine the patent perspective with the multivariate analysis method and attempting to construct a decision support method using patent analysis as the base and providing the information required for the formulation of technology policy. We select 4G LTE industry as an example, specifically the patenting activities of the 4G LTE technology field in United States, Canada, China, Japan, Korea, Sweden, Finland, France, Taiwan and Denmark to explain how to the decision support method that we have constructed in the formulation of future development strategies for national $4 \mathrm{G}$ LTE industries.

\section{Research method}

\subsection{Research Samples and Data Source}

The 4G LTE technologies focus on wireless resource allocation, signal transmission, synchronization and electronic digital data processing, 
etc. 4G LTE is a rather new and emergent technology field, first-mover and the followers all came into this field in recent 10 years. Rapid growth of patents emerges in 4G LTE field and there is abundance of data to support our empirical study. Amid the competition in the $4 \mathrm{G}$ LTE technologies, a majority of enterprises choose to use patent applications as the main method of protecting R\&D results. They can both acquire the revenue generated by $R \& D$ activities with exclusive protection and rely on their patent portfolios as a tool for patent litigations to hinder a competitor's actions. The research results can be applied to clarify patent portfolio trends in each country to assist policy-makers in understanding current trends in technology development. Furthermore, policy makers can employ the research results as the foundation of technology policy formulation and set up the 4G LTE industry's technical portfolio.

This study chose patents that were approved by the United States Patent and Trademark Office (USPTO) between January 1976 and March 2014 as research samples for several reasons. First, the USPTO has constructed a fairly comprehensive patent data searching system that allows researchers to rapidly obtain required patent information by issuing retrieval instructions, which significantly reduces the costs of gathering information. Second, the United States holds the leading position in 4G LTE technology development. The United States not only has the world's largest 4G LTE application market but also issues tens of thousands of patents annually. Thus, we chose the USPTO as the source of our data to assess the 4G LTE technology research capabilities of each country.

\subsection{Research methodology}

In the design of our research methodology, we followed steps that included patent data aggregation and confirmation as well as the calculation of each country's comparative advantage indicator using multidimensional scaling (MDS) to reduce the dimensions of analysis while clarifying each nation's technology positioning and technical expertise.

\subsubsection{Patent Data Aggregation and Confirmation.}

In this stage, we searched the IPC for LTE-related technology patents. We chose to search the USPTO, and the patents are approved between January 1976 and March 2014. This study involved searching using the IPC criteria, titles, abstracts, investors, assignees and claims to obtain the number of patents granted in 4G LTE domain as the basis for subsequent analysis.

4G LTE technology as an emergent technology, IPC of these patents do not have a concrete boundary.
Therefore, we conducted a keyword search on title, abstract and claims by "long term evolution" and LTE. Most of the 4G LTE technologies contain "long term evolution" or LTE in their claims. Using keyword search may not that exactly recruit all $4 \mathrm{G}$ LTE related patents, but the search results contain around $70 \%$ of $4 \mathrm{G}$ LTE related patents. Finally, we got 6653 patents. We further analyze the top 10 patent assignee country and top 10 classifications of IPC. We applied the Strasbourg Agreement concerning the IPC published by the World Intellectual Property Organization (WIPO) as a guideline. The Strasbourg Agreement was established for the determination of IPC. By virtue of a formal classification system, technologies are assigned unified coding and classification. A hierarchical classification method that categorizes according to section, subsection, class, subclass, group and subgroup is applied to classify and code technologies as a basis for further patent analysis. This study adopted the eighth edition of the IPC guidelines, which were issued in 2008. After reviewing the classification system, we found that $4 \mathrm{G}$ LTE technology is classified under section $\mathrm{H}$ (electricity) and $\mathrm{G}$ (Physics).

\subsubsection{Calculating the Comparative Advantage Indicator for Each Country}

Although the number of patents in the $4 \mathrm{G}$ LTE field may partially reflect each nation's R\&D capability, the findings in patent numbers alone cannot be applied to represent each country's fields of expertise in these technologies. In general, exploring the comparative technical strength of each nation requires field experts to evaluate relevant patent documents to determine the country's category in the technology classification. We applied the revealed patent advantage (RPA) analytic method, which was proposed by [32]. Prior studies have applied RPA as their analysis targets to evaluate patent value [33]. In this study, we converted the number of approved patents from each nation into the comparative advantages/disadvantages in a specific technological field. We performed this calculated by applying the following formula:

$\mathrm{RPA}_{i j}=100 \tanh \ln \left[\left(P_{\mathrm{ij}} / \sum_{i} P_{i j}\right) /\left(\sum_{j} P_{i j} /\right.\right.$

$\left.\left.\sum_{i} \sum_{j} P_{i j}\right)\right]$

Where $\mathrm{P}_{i j}$ is the number of patents of the $i^{\text {th }}$ patent classification of the $j^{\text {th }}$ country, and $\sum_{i} P_{i j}$ denotes the total number of patents of the $j^{\text {th }}$ country. Therefore, the value of $P_{i j} / \sum_{i} P_{i j}$ represents the importance of the $i^{\text {th }}$ patent classification to the $j^{\text {th }}$ 
country. $\sum_{j} P_{i j}$ denotes the total number of patents of the $i^{\text {th }}$ patent classification, whereas $\sum_{i} \sum_{j} P_{i j}$ denotes the total number of patents. Therefore, $\sum_{j} P_{i j} /$ $\sum_{i} \sum_{j} P_{i j}$ represents the importance of the $i^{\text {th }}$ patent classification to the technology industry. $\left[\left(P_{\mathrm{ij}} / \sum_{i} P_{i j}\right)\right.$ $\left./\left(\sum_{j} P_{i j} / \sum_{i} \sum_{j} P_{i j}\right)\right]$ is the comparative importance of the $i^{\text {th }}$ patent classification to the $j^{\text {th }}$ country based on the overall patent distribution in the industry.

After the calculation of RPA, we found significant differences in the comparative advantages of each country in different technology domains; thus, it may be difficult to avoid generating extreme maximum or minimum values that may lead to biases in the empirical results. We applied a ln function to process extreme values and then utilized a tanh function to convert the comparative advantage indicator of each patent to a value between 1 and -1 . Finally, the calculated result was multiplied by 100 , such that the resulting RPA value is between -100 and 100. If an RPA value is positive (negative), then this result indicates that a nation has comparative advantages (disadvantages) in a specific field. Additionally, if the difference of the RPA values in a particular technical domain is greater than 15 , then there is a statistically significant comparative advantage between two countries in that particular technical domain.

\subsubsection{Using Multidimensional Scaling (MDS) to Reduce the Dimensions for Analysis}

Some studies use cluster analysis to grouping analytical targets [34, 35], but clustering analysis cannot reduce dimensions to represent data in a more comprehensive way. In this study, we focused on representing the reduced technological dimensions of 4G LTE fields. After examining the technical domains related to $4 \mathrm{G}$ LTE, we used the IPC to identify 12 main related technical fields. These 12 technical fields in different countries have increased the difficulty of data processing and may have distracted us from the focus of our research. Thus, we applied the parsimony principle to reduce indicators with the expectation of using fewer dimensions to present the relative advantage of each country in the 4G LTE technical domains. These technical domains were shown in MDS maps, which represent the distance between any two technical domains. By reading the MDS map, we can tell the related technological development.

3.2.4. Clarifying Each Nation's Technology Positioning and Analyzing Its Technical Expertise
Using the MDS results of the top ten 4G LTE assignee's nations (United States, China, Japan, Korea, France, Taiwan, Denmark, Sweden, Finland and Canada), we identified the location of each nation's technology positioning in two-dimensional space. Following these positioning results, we were able to sketch a similarity perceptual map for each nation's 4G LTE technology development to assist us in understanding the positioning and layout of each country's 4G LTE technical domains. Additionally, we attempted to create a 4G LTE technology positioning diagram and to summarize technology similarity classifications based on each nation's relative capability in different technology classifications. We also considered the similarity between the technical capabilities of these countries and provided a visual representation method to represent the competitive/cooperative relationships among the nations with respect to the $4 \mathrm{G}$ LTE technologies.

\section{Analysis Results}

This study conducted a search of the granted $4 \mathrm{G}$ LTE patents owned by United States, Canada, Japan, China, Korea, Denmark, Sweden, Finland, France and Taiwan. The search results are displayed in Table 2 . In terms of the total number of patents in the $4 \mathrm{G}$ LTE field, United States has a leading position with 2845 patents, followed by Korea with 1028 patents and Japan has 760 4G LTE patents. Top 10 4G LTE patent's assignee country are listed in Table 1.

According to this table, we can briefly conclude that in 4G technologies, France, Taiwan and Denmark own less patents in $4 \mathrm{G}$ technologies. If we further investigate the number of patents owned and the RPA values of top 12 technical fields of each country based on IPC, then we can assess each nation's comparative advantages in specific technology fields. Using RPA analysis, we can determine each country's development status in 12 4G LTE technical domains, which can serve as the basis for allocating R\&D resources and formulating technology policy. Through RPA analysis, we can also identify the technical fields where each nation has comparative advantages (as shown in Table 2). In the analysis of technology positioning, we initially applied multidimensional scaling to reduce the number of dimensions; we sought to reduce $124 \mathrm{G}$ LTE technical domains to a small number of dimensions to simplify the data. As a newly developed technical field, 4G LTE patents are rather fewer than other well-developed technological fields. 4G LTE patents mainly focused on one technical field, H04W. 
Table 1 Top 10 4G LTE patent assignees' country and the amount of patents

\begin{tabular}{lrlr}
\hline Country & Total patents & Country & Total patents \\
\hline United States & 2,845 & China & 275 \\
Korea & 1,028 & Canada & 185 \\
Japan & 760 & France & 158 \\
Sweden & 524 & Taiwan & 106 \\
Finland & 397 & Denmark & 89 \\
\hline
\end{tabular}

Table 2. Countries Involved in the 4G LTE Technology Category and Patents Approved / RPA Value of Technology Categories

\begin{tabular}{|c|c|c|c|c|c|c|c|c|c|c|}
\hline & \multicolumn{2}{|c|}{ United States } & \multicolumn{2}{|c|}{ Canada } & \multicolumn{2}{|c|}{ Japan } & \multicolumn{2}{|c|}{ Korea } & \multicolumn{2}{|c|}{ China } \\
\hline & Patents & RPA & Patents & RPA & Patents & RPA & Patents & RPA & Patents & RPA \\
\hline $\mathrm{H} 04 \mathrm{~W}$ & 808 & -12.9962 & 67 & 8.4591 & 330 & $25.5959^{*}$ & 375 & 6.4257 & 107 & 13.8927 \\
\hline H04B & 521 & 3.4470 & 38 & $12.1088^{*}$ & 148 & 6.3810 & 194 & 0.9274 & 49 & -3.7163 \\
\hline H04L & 351 & -1.08664 & 14 & -48.3262 & 76 & -24.7708 & 129 & -4.9118 & 39 & 8.3976 \\
\hline H04J & 200 & -15.3598 & 11 & -33.6269 & 61 & -5.4263 & 120 & 28.8596 & 38 & $44.3606^{*}$ \\
\hline H04M & 224 & $29.0847^{\#}$ & 9 & -20.6551 & 34 & -28.9339 & 49 & -25.2098 & 10 & -47.5617 \\
\hline H04K & 45 & -0.2685 & 4 & 27.5043 & 15 & 18.4515 & 11 & -42.0887 & 6 & 26.7972 \\
\hline $\mathrm{H} 04 \mathrm{~N}$ & 24 & 9.1458 & 0 & N/A & 8 & $27.3599^{\#}$ & 13 & $41.4465^{* \#}$ & 0 & N/A \\
\hline $\mathrm{H} 03 \mathrm{M}$ & 62 & 24.6831 & 12 & $86.5578^{* \#}$ & 6 & -66.1451 & 19 & 3.2012 & 2 & -71.1239 \\
\hline H01Q & 24 & -2.1913 & 1 & -45.7864 & 6 & -11.9684 & 5 & -55.6570 & 1 & -72.0029 \\
\hline G01R & 46 & 5.8835 & 3 & 3.4225 & 6 & -59.8001 & 13 & -23.7485 & 8 & $53.8449^{* \#}$ \\
\hline G06F & 272 & 24.6015 & 12 & -16.2900 & 30 & -78.9820 & 54 & -38.2486 & 2 & -98.2647 \\
\hline G08C & 26 & -13.5197 & 3 & 38.8346 & 8 & -2.6728 & 12 & 5.3412 & 1 & -80.1166 \\
\hline \multirow[t]{3}{*}{ Total } & 2603 & & 174 & & 718 & & 994 & & 263 & \\
\hline & \multicolumn{2}{|c|}{ Sweden } & \multicolumn{2}{|c|}{ Finland } & \multicolumn{2}{|c|}{ France } & \multicolumn{2}{|c|}{ Taiwan } & \multicolumn{2}{|c|}{ Denmark } \\
\hline & Patents & RPA & Patents & RPA & Patents & RPA & Patents & RPA & Patents & RPA \\
\hline H04W & 182 & 1.6625 & 112 & -12.5010 & 54 & 5.1362 & 36 & -0.2297 & 31 & 11.5912 \\
\hline H04B & 94 & -4.0067 & 61 & -12.8579 & 13 & -64.6132 & 17 & -14.7521 & 14 & -7.4360 \\
\hline H04L & 103 & 38.0769 & 43 & -12.8639 & 34 & $49.4798^{* \#}$ & 12 & -14.6243 & 9 & -16.5190 \\
\hline H04J & 51 & 11.6043 & 36 & 11.1017 & 4 & -82.7186 & 8 & -13.3451 & 4 & -50.7335 \\
\hline H04M & 15 & -64.4727 & 12 & -56.9073 & 13 & $32.7906^{*}$ & 8 & 20.3838 & 5 & 0.4994 \\
\hline $\mathrm{H} 04 \mathrm{~K}$ & 11 & 22.2639 & 4 & -41.5264 & 2 & -22.4618 & 2 & 12.2626 & 3 & $66.2343^{*}$ \\
\hline $\mathrm{H} 04 \mathrm{~N}$ & 1 & -89.5447 & 3 & -0.6932 & 0 & N/A & 0 & N/A & 1 & 39.7831 \\
\hline $\mathrm{H} 03 \mathrm{M}$ & 4 & -69.1551 & 2 & -83.3915 & 2 & -75.6257 & 2 & -56.1932 & 1 & -35.1705 \\
\hline H01Q & 1 & -91.5758 & 2 & -48.2107 & 0 & N/A & 12 & $98.7247^{* \#}$ & 4 & $93.4659^{\#}$ \\
\hline G01R & 12 & 33.9079 & 4 & -38.1955 & 4 & 46.5447 & 3 & 51.4125 & 0 & N/A \\
\hline G06F & 22 & -55.5091 & 74 & $73.0840^{* \#}$ & 19 & 44.4231 & 2 & -89.0024 & 6 & -5.5109 \\
\hline G08C & 10 & $49.7773^{* \#}$ & 6 & 36.1604 & 1 & -46.7167 & 1 & -15.3458 & 0 & N/A \\
\hline Total & 506 & & 359 & & 145 & & 102 & & 78 & \\
\hline
\end{tabular}

Note: * Strongest performing country within the category; \# Strongest performing specific technical category of a country

The dimension scree plot result indicates that there are four factors that eigenvalue more than 1 . We extracted the largest two factors, which explain 50\% of data variance. According to the factor analysis results, we conduct the MDS analysis of both 12 technical fields and 10 countries. Second, we estimated the coordinates of the technology positioning of each country on a two-dimensional space. Meanwhile, based on computation results, we developed a similarity perceptual map of $4 \mathrm{G}$ LTE technology development in United States, Canada,
China, Japan, Korea, Finland, France, Taiwan and Denmark to render each nation's technological portfolio in the 4G LTE field (as shown in Figure 1). Finally, we estimated and created a perceptual similarity map of the 12 4G LTE technology fields with technology positioning coordinates on a twodimensional space. Employing each country's comparative capability in different technology classifications, we determined a number of closely related technical fields that was sufficient to 


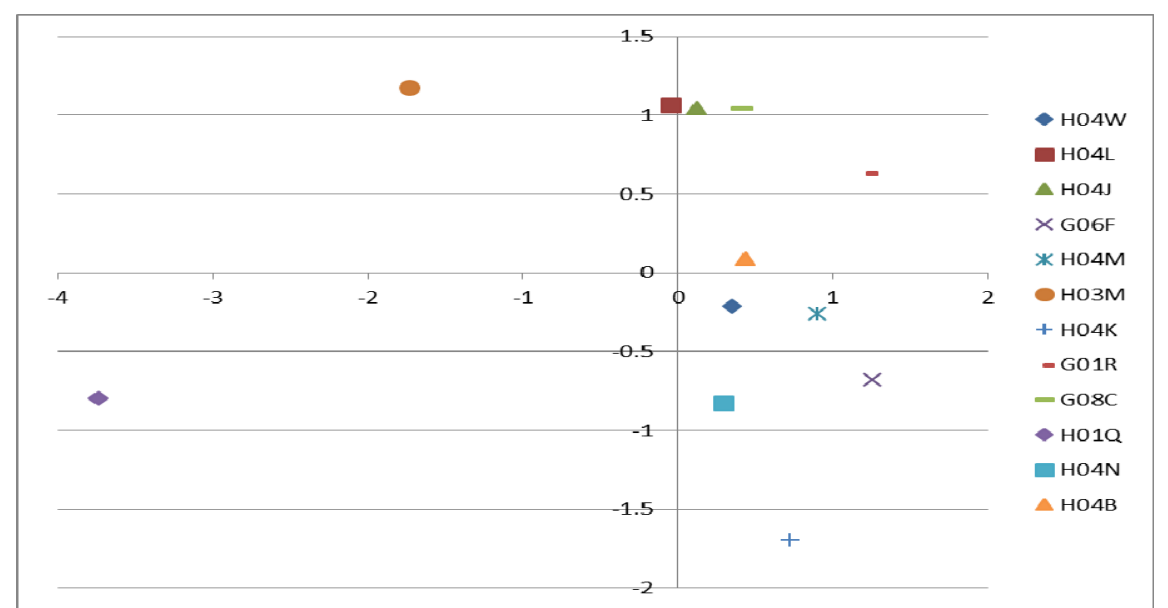

Figure 1 Technological positioning of MDS

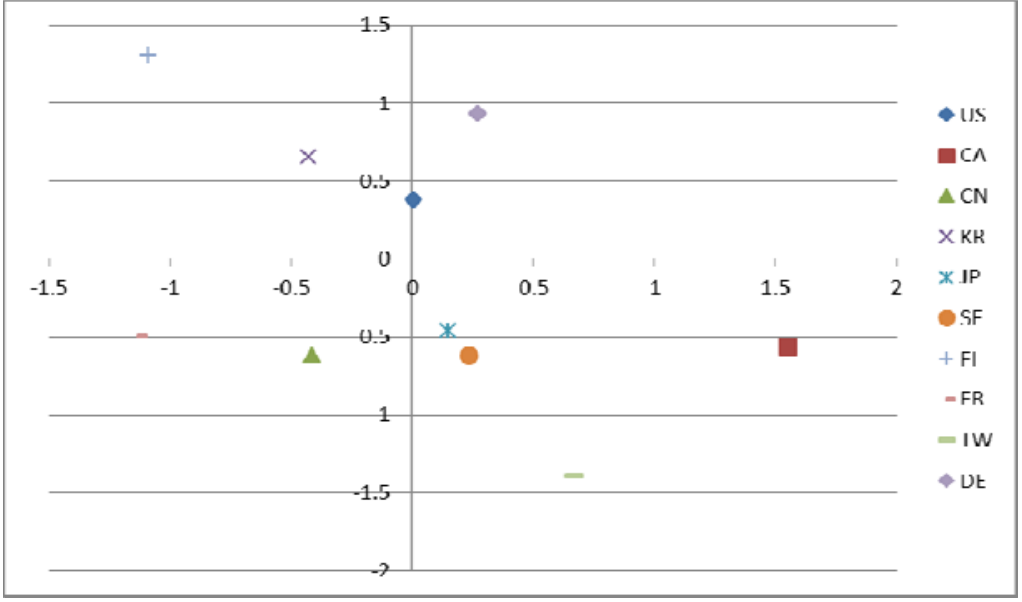

Figure 2 Distribution for 4G LTE technology positioning of each country

subsequently explore each nation's advantageous technical domain (as shown in Figure 2).

This study also included the MDS method to explore the comparative advantages of each nation's 4G LTE technology domain that policy-makers may use to formulate technology policy. According to the 4G LTE technical expertise of each country, we grouped the technologies based on each country's technical strength in these domains. Several MDS methods are applied in calculating the dissimilarity/ similarity among the countries and technological fields. By distinguishing by $\mathrm{x}$-axis and $\mathrm{y}$-axis, both countries and technological fields can be categorized into four groups. Thus, we chose to use four groups for our analysis. To further explore the implications of the four groups, this study attempted to use the expert interview and literature review modes of analysis to generate the strategic implications of the four groups. We divided the 12 LTE technical fields into the following components: a manufacturing group, an assembly group, a material design group and a component processing technology group.
Group 1: Measurements of the strength of communication signal variation. The identification of communication system connections. G01R, G08C, $\mathrm{H} 04 \mathrm{~J}$ and $\mathrm{H} 04 \mathrm{~B}$ are categorize into this group. These patents are related to the digital signal transmission and communication in $4 \mathrm{G}$ spectrum.

Group 2: Transmit content packages encoding, which includes the confirmation of encoding, decoding packages and the information correctness. Patents in H03M and H04L are classified in this group. This group is related to data packages and data transmission, make sure that the encoding and decoding processes are corrected. The receiver will get correct information from information sender.

Group 3: 4G antenna signal receiving. Group 3 is also a single classification group. H01Q is the main patent classification in this group. Patents are related to the antenna data receiving technologies are categorized in this group.

Group 4: 4G application technologies. This group includes video, audio signals' wireless transmission. Patents are related to decoding and encoding of 
signals can be categorized in this group, including H04W, H04M, H04N, G06F, and H04K.In Table 2, we listed the advantageous technical domains of each country. United States owned the largest patent pool and the widest technical domains, but the RPAs of each field does not become the leader of technology development. The largest RPA of United States is H04M. Canada does not develop 4G LTE technologies in $\mathrm{H} 04 \mathrm{~N}$, but it is the leader of H03M, both across 10 countries and in its own country. Japan stands the most advantageous technological development position in field H04W. H04N is the most advantageous field of Korea, as well as the most advantageous of technical fields in Korea's 4G LTE technology development. China has the prominent advantageous in field G01R, both in its own technological development and across the 10 countries. Sweden has the most advantageous technological field in G08C. Finland has the most advantageous technological field in G06F. H04L is the most advantageous field of France. Taiwan has the most advantageous in H01Q. H01Q is the most advantageous field in Denmark's technological development of 4G LTE field.

\section{Discussion and Conclusion}

\subsection{Discussion}

The contributions of this study lie in two areas: supporting the formulation of national technology policy and improving methods of technology positioning. In terms of supporting the formulation of national technology policy, we employed patent data as the foundation and attempted to propose a decision support system that integrates MDS methods. With the assistance of computation and analysis, we created technology positioning maps to display a variety of information that may be required for policy formulation in a visual manner to enable policymakers to comprehend the trajectories of development and the evolutionary processes of the specific technology of their nation and other nations [31]. Technological evolution is the main concern in practical and academia fields [36]. In this study, we provided an integration method which combining patents and multivariate analytical method to analyze 4G LTE technological development. This method provides the visualization information for decision makers to consider objective criteria, which reflects the insights from [31]. By doing so, we can enhance the efficiency and efficacy of the usage of technology roadmap. Furthermore, we can forecast future technological development trends, offer sufficient and credible evidence as the basis for policy formulation, and assist governments in clarifying future technological development directions. The quality of technology governance is naturally enhanced $[9,14]$.

In response to the trend of annually declining $R \& D$ budgets, governments should attempt to construct a set of technology monitoring mechanisms. By examining current data (e.g., patents and literature), governments can understand current trends in the development of the technology industry and thus identify technical fields that are marked for future development. Limited R\&D resources can be allocated to the fields with the greatest development potential to create maximum benefit from the resources and to respond to questions of accountability with which the public and the legislature are concerned. The quantitative measures provide objective method to describe the data we collect and MDS can reduced the dimensions [28]. In turn, such clarification and evidence may convince stakeholders to support technology policy proposed by the government and encourage consensus on the future development of the country [11, 24].

We believe that analyzing a country's strengths can assist in examining its 4G LTE technology portfolio and its international competition status; using these data, policy-makers can develop competitive or cooperative policies. In terms of designing competitive policy, policy-makers may formulate strategic positioning from the technology positioning map while simultaneously considering how to respond to the actions of competitors. In terms of cooperative policy, policy-makers can determine which countries have complementary technology by consulting the positioning map. With the assistance of strategic alliances, patent licensing and/or collaborative research, a nation's gap in $R \& D$ capabilities may be complemented to enable improvements and upgrading of technological innovation, thus achieving the goal of enhancing international competitiveness.

In terms of improving the method of technology positioning, this study offers the following implications. First, we aimed to respond to the suggestions of previous researchers to combine patent analysis and multivariate methods in an attempt to obtain analysis results with greater reliability and validity in support of the formulation of technology policy by the government. Second, this study also attempted to offer a new technology monitoring system to assist researchers or policymakers in surveying development in the technology industry based on patent information. This monitoring system can provide visual information and assist policy-makers in mapping the technology development. Third, we believe that the combination 
of the patent information and multivariate analytic methods should be more effective in the process of policy formulation than either method would perform independently. Finally, we offer a detailed operating procedure to explain data processing procedures and operating methods. This study aims to share this $4 \mathrm{G}$ LTE experience with countries in need and to promote the formulation of quality technology policy or use this information as a basis for more advanced discussions.

\subsection{Limitations and Future Research}

Several issues in this study have yet to be clarified. First, we believe that different types of data (e.g., patents, literature and expert opinions) and different research methods (e.g., multivariate, social network or bibliometric analysis) have unique assumptions, functions, applicable timing, strengths, weaknesses and limitations. In addition to combining patent data and multivariate methods of analysis, researchers should attempt to apply different types of data and/or different research methods to explore technology positioning issues from different perspectives and to provide government with ample information as a reference in the formulation of technology policy. Integrating data from both patent database and bibliometric data may bring new insights for further analysis.

Second, this study chose patent data approved by the USPTO as the basis for its data searches, using the largest 4G LTE technology market in the world as the data source. We applied keyword search from abstract and claims to obtain analytical data. By doing so, it will cause some data missing patents. Will the results be similar if the same method is applied to other patent offices? We suggest that future researchers may consider including this issue in their research using other indicators or methods to obtain results that better reflect each nation's research energy capabilities.

Third, because applying for and obtaining patents is an effective way for high-tech industries to protect their intellectual property rights, we chose to use patent data to explore the trajectory of each country's 4G LTE technology development. Reviewing academic publication records can also be a feasible method for analyzing the status of each nation's technology development. This study suggests that researchers may adopt the perspective of bibliometric by consider the publications published by companies, universities and research institutes in the 4G LTE technology domains in subsequent exploration.

Fourth, besides technology flow of 4G LTE technologies, this study did not take market mechanism and time into consideration (Kim 1998).
Each country faces different in their domestic market, but they all need to compete with other countries. The RPA can be one of the competitive advantages a country has to obtain the leading position in global 4G LTE market.

We applied RPA indicators as a measure of each nation's comparative advantages in the 4G LTE technology domains to evaluate each country's R\&D capabilities. In essence, RPA indicators represent a relative concept rather than an absolute concept. We suggest that researchers who use our results as the basis of their studies should consider and adjust the application of RPA to ensure that their results truly reflect each nation's comparative advantages in technical domains.

\section{References}

[1] Brown, W. B., and D. Gobeli, "Observations on the measurement of R\&D productivity: A case study," IEEE Transactions on Engineering Management, 39(4), 1992, pp. 325-331.

[2] Kostoff, R. N., "Research requirements for research impact assessment," Research Policy, 24(6), Nov, 1995, pp. 869-882.

[3] Dohse, D., "Technology policy and the regions-The case of the BioRegio contest," Research Policy, 29(9), Dec, 2000, pp. 1111-1133.

[4] Chang, P.-L., and C.-W. Hsu, "The development strategies for Taiwan's semiconductor industry," IEEE Transactions on Engineering Management, 45(4), Nov, 1998, pp. 349-356.

[5] Carayannis, E. G., and P. Laget, "Transatlantic innovation infrastructure networks: public-private, EU-US R\&D partnerships," R\&D Management, 34(1), 2004, pp. 17-31

[6] Lai, H. C., and Y. C. Liaw, "Evaluating the effects of innovation policies on Taiwan's IC design industry: a fuzzy integral MCDM approach," International Journal of Technology, Policy and Management, 7(4), 2007, pp. 396421.

[7] Jaffe, A. B., "The importance of spillovers in the policy mission of the advanced technology program," The Journal of Technology Transfer, 23(2), 1998, pp. 11-19.

[8] Wang, Y., N. Roijakkers, and W. Vanhaverbeke, "Learning-by-Licensing: How Chinese Firms Benefit From Licensing-In Technologies," IEEE Transactions on Engineering Management, 60(1), Feb, 2013, pp. 46-58.

[9] Debackere, K., and W. Glänzel, "Using a bibliometric approach to support research policy making: The case of the Flemish BOF-key," Scientometrics, 59(2), 2004, pp. 253-276.

[10] Huang, C. Y., J. Z. Shyu, and G. H. Tzeng, "Reconfiguring the innovation policy portfolios for Taiwan's SIP Mall industry," Technovation, 27(12), Dec, 2007, pp. 744-765.

[11] Lee, L. C., Y. Y. Lee, and Y. C. Liaw, "Bibliometric analysis for development of research strategies in 
agricultural technology: The case of Taiwan," Scientometrics, 93(3), Dec, 2012, pp. 813-830.

[12] Daim, T. U., G. Rueda, H. Martin, and P. Gerdsri, "Forecasting emerging technologies: Use of bibliometrics and patent analysis," Technological Forecasting and Social Change, 73(8), 2012, pp. 981-1012.

[13] Chen, Y. H., C. Y. Chen, and S. C. Lee, "Technology forecasting of new clean energy: The example of hydrogen energy and fuel cell," African Journal of Business Management 4(7), Jul 4, 2010, pp. 1372-1380.

[14] Grupp, H., and H. A. Linstone, "National technology foresight activities around the globe: Resurrection and new paradigms," Technological Forecasting and Social Change, 60(1), Jan, 1999, pp. 85-94

[15] Chuang, Y. W., L. C. Lee, W. C. Hung, and P. H. Lin, "Forging into the innovation lead-A comparative analysis of scientific capacity," International Journal of Technology, Policy and Management, 14(3), 2010, pp. 511-529.

[16] Teixeira, A. A. C., and L. Mota, "A bibliometric portrait of the evolution, scientific roots and influence of the literature on university-industry links," Scientometrics, 93(3), 2012, pp. 719-743.

[17] Ernst, H., "The use of patent data for technological forecasting: The diffusion of CNC-technology in the machine tool industry," Small Business Economics, 9(4), Aug, 1997, pp. 361-381.

[18] Cheng, A. C., C. J. Chen, and C. Y. Chen, "A fuzzy multiple criteria comparison of technology forecasting methods for predicting the new materials development," Technological Forecasting and Social Change, 75(1), Jan, 2008, pp. 131-141.

[19] Hekkert, M. P., R. A. A. Suurs, S. O. Negro, S. Kuhlmann, and R. E. H. M. Smits, "Functions of innovation systems: A new approach for analysing technological change," Technological Forecasting and Social Change, 74(4), May, 2007, pp. 413-432.

[20] Hsu, C. W., and H. C. Chiang, "The government strategy for the upgrading of industrial technology in Taiwan," Technovation, 21(2), Feb, 2001, pp. 123-132.

[21] Martinsons, M. G., "Hong Kong government policy and information technology innovation: the invisible hand, the helping hand, and the hand-over to China," IEEE Transactions on Engineering Management, 45(4), Nov, 1998, pp. 366-380.

[22] Cozzarin, B. P., "Performance measures for the socioeconomic impact of government spending on R\&D," Scientometrics, 68(1), 2006, pp. 41-71.

[23] Teece, D. J., "Firm organization, industrial structure, and technological innovation," Journal of Economic Behavior \& Organization, 31(2), Nov, 1996, pp. 193-224.

[24] Hill, J. R., and J. Thies, "Program theory and logic model to address the co-occurrence of domestic violence and child maltreatment," Evaluation and Program Planning, 33(4), Nov, 2010, pp. 356-364.

[25] Kim, B., and H. Oh, "An effective R\&D performance measurement system: survey of Korean R\&D researchers," OMEGA-International Journal of Management Science, 30(1), 2002, pp. 19-31.

[26] Marinova, D., and P. Newman, "The changing research funding regime in Australia and academic productivity," Mathematics and Computers in Simulation, 78(2-3), Jul, 2008, pp. 283-291.

[27] Dolfsma, W., and L. Leydesdorff, "Innovation systems as patent networks: The Netherlands, India and nanotech," Innovation: Management, Policy \& Practice, 13(3), 2011, pp. 311-326.

[28] Suominen, A., "Analysis of technological progression by quantitative measures: a comparison of two technologies," Technology Analysis \& Strategic Management, 25(6), Jul 1, 2013, pp. 687-706.

[29] Kim, C., and M.-S. Kim, "Identifying core environmental technologies through patent analysis," Innovation: Management, Policy \& Practice, 17(1), Feb, 2015, pp. 139-158.

[30] Van Dijk, J. W. A., "Foresight studies: A new approach in anticipatory policy making in the Netherlands," Technological Forecasting and Social Change, 40(3), 1991, pp. 223-234.

[31] Yoon, B., and R. Phaal, "Structuring technological information for technology roadmapping: data mining approach," Technology Analysis \& Strategic Management, 25(9), Oct 1, 2013, pp. 1119-1137.

[32] Schmoch, U., "Evaluation of technological strategies of companies by means of MDS maps," International Journal of Technology, Policy and Management, 10(4-5), 1995, pp. 426-440.

[33] Tseng, F., C. Hsieh, Y. Peng, and Y. Chu, "Using patent data to analyze trends and the technological strategies of the amorphous silicon thin-film solar cell industry," Technological Forecasting and Social Change, 78(2), 2011, pp. 332-345.

[34] Hung, S.-C., N.-C. Liu, and J.-B. Chang, "The taxonomy and evolution of technology strategies: a study of Taiwan's high-technology-based firms," IEEE Transactions on Engineering Management, 50(2), 2003, pp. 219-227.

[35] Martínez-Torres, M. R., and S. L. Toral, "International comparison of R\&D investment by European, US and Japanese companies," International Journal of Technology Management, 49(1/2/3), 2010, pp. 107-122.

[36] Clarke, K., "Pathways to technology strategy: Technological configurations, stability and change," Technology Analysis \& Strategic Management, 4(1), 1992, pp. 33-49.

\section{Acknowledgement}

This research was supported by Taiwan Ministry of Science and Technology, project no.102-2410-H-131 -002. We thank Te-Yi Chan, associate researcher, from Science \& Technology Policy Research and Information Centre, National Applied Research Laboratories, who provided insight and expertise in patent search that greatly assisted this research. 